\title{
On a problem of Alfréd Rényi
}

\author{
by
}

Pelegrí Viader, Lluís Bibiloni and Jaume Paradís (Barcelona)

1. Introduction. Every irrational number in $(0,1)$ can be uniquely expanded into an Engel series

$$
x=\frac{1}{q_{1}}+\frac{1}{q_{1} q_{2}}+\ldots+\frac{1}{q_{1} q_{2} \ldots q_{n}}+\ldots
$$

where the $q_{i}$ are positive integers satisfying $2 \leq q_{1} \leq q_{2} \leq \ldots$ We denote the series in (1) by $\left\langle q_{1}, q_{2}, \ldots\right\rangle_{\mathrm{E}}$. Erdős, Rényi and Szüsz [2] studied the metrical properties of Engel's expansions completing the results announced by Borel [1] and Lévy [6].

The alternated version of Engel's series is known as Pierce expansion: for an irrational $x$ in $(0,1]$ we have the unique expansion

$$
x=\frac{1}{q_{1}}-\frac{1}{q_{1} q_{2}}+\ldots+\frac{(-1)^{n+1}}{q_{1} q_{2} \ldots q_{n}}+\ldots
$$

In this case, the $q_{n}$ are positive integers satisfying $1 \leq q_{1}<q_{2}<\ldots$ We denote the right-hand side of $(2)$ by $\left\langle q_{1}, q_{2}, \ldots\right\rangle_{\mathrm{P}}$. The $q_{n}$, in both Engel and Pierce cases, are called elements or partial quotients.

Both expansions were studied by Sierpiński [14]. The metrical theory of Pierce expansions was studied by Shallit [12]. More recent contributions to the subject are found in $[7,8,11,13]$.

In the first part of a 1962 paper [9], Rényi provided a shorter way of reaching most of the results of [2]. Of these, the more spectacular is initially due to Borel [1], and states that for almost all $x \in(0,1]$, the elements of their Engel series satisfy

$$
\lim _{n \rightarrow \infty} \sqrt[n]{q_{n}(x)}=e
$$

In [12] Shallit proved that the same holds for Pierce expansions. These (and other) coincident results indicate that the growth of the sequence of elements

1991 Mathematics Subject Classification: 11J70, 11K55.

Key words and phrases: Pierce expansions, Engel's series, metric theory. 
of Engel's series and Pierce expansions is about the same (with measure 1). This had already been noted by Rényi and by Williams [15].

In the second part of [9], Rényi proved for Engel's series that:

(i) For almost all $x$ the sequence $q_{n}(x)$ is strictly increasing for $n \geq$ $n_{0}(x)$.

(ii) The probability of $q_{n}(x)$ being strictly increasing from the very beginning is exactly $1 / 2$.

The first problem cannot be directly translated into Pierce expansions as the sequence of partial quotients $q_{n}$ is already strictly increasing. Nevertheless, we can ask about the measure of the set of those $x$ at which the "jump" between two consecutive elements is greater than a fixed positive integer $k$. Of course, this generalization also applies to Engel's series. Galambos [4, p. 298] proved that for almost all $x \in(0,1]$,

$$
\lim _{n \rightarrow \infty} \frac{\log \left(q_{n}-q_{n-1}\right)}{n}=1,
$$

a result that obviously implies the generalization of Rényi's first problem.

Rényi's problem (ii) in its original setting is easy to solve, but its generalization is not so easy to tackle. That is the aim of the present article: to extend result (ii) above, for both Engel and Pierce cases, by computing the exact measure of the set of all $x$ at which the jump between consecutive elements is greater than or equal to $k$ from the very beginning (Section 4).

In Section 2 we summarize some results on Pierce and Engel series, and Section 3 consists of two lemmas needed in the following.

2. Some metrical results. The results of this section alongside with their proofs can be found in $[2,12]$. All values $x$ will always refer to real numbers in $(0,1]$, and $\boldsymbol{\lambda} A$ will denote the Lebesgue measure of the set $A \subset$ $[0,1]$.

Given a finite sequence of positive integers of length $r, a_{1}, \ldots, a_{r}$, satisfying $a_{1}<\ldots<a_{r}$ in the case of Pierce and $2 \leq a_{1} \leq \ldots \leq a_{r}$ in the case of Engel (admissible or realizable sequences), a cylinder of rank $r$ is the set

$$
C_{r}\left(a_{1}, \ldots, a_{r}\right)=\left\{x: q_{1}(x)=a_{1}, \ldots, q_{r}(x)=a_{r}\right\} .
$$

In the case of Pierce expansions, cylinders are intervals with endpoints $\left\langle a_{1}, \ldots, a_{r}\right\rangle_{\mathrm{P}}$ and $\left\langle a_{1}, a_{2}, \ldots, a_{r}+1\right\rangle_{\mathrm{P}}$ taken in proper order, and length

$$
\left|C_{r}\left(a_{1}, \ldots, a_{r}\right)\right|=\frac{1}{a_{1} \ldots a_{r}\left(a_{r}+1\right)} .
$$

$(|I|$ denotes the length of the interval $I$. 
For Engel's case, cylinders are intervals with endpoints $\left\langle a_{1}, \ldots, a_{r}\right\rangle_{\mathrm{E}}$ and $\left\langle a_{1}, \ldots, a_{r}-1\right\rangle_{\mathrm{E}}$ and length

$$
\left|C_{r}\left(a_{1}, \ldots, a_{r}\right)\right|=\frac{1}{a_{1} \ldots a_{r}\left(a_{r}-1\right)} .
$$

The sets with which the metrical theory concerns itself are, in general, expressed as unions of disjoint cylinders. In the case of Pierce expansions, this computation depends on the value of sums of the type

$$
A_{r}(m, n)=\sum_{m \leq q_{1}<\ldots<q_{r} \leq n} \frac{1}{q_{1} \ldots q_{r}},
$$

where $m$ and $n$ are two positive integers $(m<n)$ and $0<r \leq n-m$. That is, the sum extends over the set of Pierce admissible sequences of length $r$ whose elements are in the range $[m, n]$. The consideration of Engel's admissible sequences gives rise to the same type of sums, where repetitions are allowed, that is, sums of the type

$$
B_{r}(m, n)=\sum_{m \leq q_{1} \leq \ldots \leq q_{r} \leq n} \frac{1}{q_{1} \ldots q_{r}} .
$$

In this case, each sum depends on $r, 0 \leq r<\infty$.

Since for fixed $m, n$ the numbers $A_{r}$ are nothing but the elementary symmetric functions for the polynomial whose roots are $\left\{\frac{1}{m}, \frac{1}{m+1}, \ldots, \frac{1}{n}\right\}$, the generating function of the complex variable $z$ for the sequence $\left\{A_{r}\right\}$ is

$$
\left(1+\frac{z}{m}\right)\left(1+\frac{z}{m+1}\right) \ldots\left(1+\frac{z}{n}\right)=A_{0}+A_{2} z^{2}+\ldots+A_{n-m+1} z^{n-m+1} .
$$

It can also be proved that, in the case of an Engel admissible sequence the generating function for $\left\{B_{r}\right\}$ is the rational function

$$
\frac{1}{\left(1-\frac{z}{m}\right)\left(1-\frac{z}{m+1}\right) \ldots\left(1-\frac{z}{n}\right)}=B_{0}+B_{1} z+B_{2} z^{2}+\ldots
$$

2.1. The shift transform. An alternative approach to the theory of Engel's and Pierce's series is based on the introduction of the shift transforms $T_{\mathrm{P}}$ and $T_{\mathrm{E}}$ of which we shall make a very limited use. This is the bridge which links these systems of representation of real numbers to ergodic theory and dynamical systems (see [3, Ch. 5] or the more recent [10]). If $x=\left\langle q_{1}, q_{2}, \ldots\right\rangle$ and if $T$ refers to either $T_{\mathrm{P}}$ or $T_{\mathrm{E}}$, the equation

$$
T\left\langle q_{1}, q_{2}, \ldots\right\rangle=\left\langle q_{2}, q_{3}, \ldots\right\rangle
$$

may be taken as the definition of each of the shift transforms. It is easy to see that the restriction of the transforms to a cylinder $C_{1}(m)$ takes the 
forms $T_{\mathrm{E}}(x)=m x-1$ and $T_{\mathrm{P}}(x)=1-m x$. Now, if $I=(x, y)$ is an open interval, $I \subset C_{1}(m)$, its image under $T$ is an interval of length

$$
|T y-T x|=m(y-x) \Leftrightarrow|T I|=m|I| .
$$

As this last equality is true for intervals it must be true for Lebesgue measurable sets. Thus for every measurable set $A$,

$$
A \subset C_{1}(m) \Rightarrow \boldsymbol{\lambda} T A=m \boldsymbol{\lambda} A \text {. }
$$

3. Some preliminary lemmas. Consider a fixed positive integer $k \geq 1$.

Lemma 1 (Pierce expansions). For positive integers $m$ and $n(m \leq n)$, let $X_{m, n}=\left\{x: q_{1}(x) \geq m\right.$ and $\exists j(j \geq 1), q_{j}(x)=n$ and $\left.q_{j+1}(x)<n+k\right\}$.

Then

$$
\boldsymbol{\lambda} X_{m, n}=\frac{1}{m}\left(\frac{1}{n+1}-\frac{1}{n+k}\right) .
$$

Proof. $X_{m, n}$ is the disjoint union of the intervals whose endpoints are

$$
\left\langle q_{1}, \ldots, q_{j-1}, n, n+1\right\rangle_{\mathrm{P}}, \quad\left\langle q_{1}, \ldots, q_{j-1}, n, n+k\right\rangle_{\mathrm{P}},
$$

for all admissible values of $q_{1}, \ldots, q_{j-1}$. Consequently, the total measure is

$$
\frac{1}{n}\left(\frac{1}{n+1}-\frac{1}{n+k}\right) \sum_{m \leq q_{1}<\ldots<q_{j-1} \leq n-1} \frac{1}{q_{1} \ldots q_{j-1}} ;
$$

setting $z=1$ in (4), this last sum is

$$
\left(1+\frac{1}{m}\right)\left(1+\frac{1}{m+1}\right) \ldots\left(1+\frac{1}{n-1}\right)=\frac{n}{m} .
$$

Thus, the measure we seek equals

$$
\frac{1}{n}\left(\frac{1}{n+1}-\frac{1}{n+k}\right) \frac{n}{m}=\frac{1}{m}\left(\frac{1}{n+1}-\frac{1}{n+k}\right) .
$$

For Engel's series we obtain:

Lemma 2 (Engel's series). For positive integers $m$ and $n(m \leq n)$, let $Y_{m, n}=\left\{x: q_{1}(x) \geq m\right.$ and $\exists j(j \geq 1), q_{j}(x)=n$ and $\left.q_{j+1}(x)<n+k\right\}$. Then

$$
\lambda Y_{m, n}=\frac{1}{m}\left(1-\frac{1}{n}\right)\left(\frac{1}{n-1}-\frac{1}{n-1+k}\right) .
$$

4. Rényi's second problem generalized. When dealing with Pierce expansions, the closest one can get to the second of Rényi's problems is to ask for the measure of the set $G$ of numbers whose partial quotients jump more than two units from the very beginning. A direct argument based on 
the inclusion and exclusion formula may be used to show that the measure of the complement of $G$ in $[0,1]$ is $1 / e$. Thus

$$
\lambda G=1-\frac{1}{e} .
$$

Besides being tedious in its details, the proof in terms of the inclusion and exclusion formula is impossible to extend to larger values of the jump $k$, so we are forced to take a different route. We give the details only for Pierce expansions as the modifications for Engel's series are trivial.

We call

$$
E_{m}^{(k)}=\left\{x: q_{1}(x) \geq m, \forall j, q_{j+1}(x) \geq q_{j}(x)+k\right\} .
$$

With this notation, the set $G$ above is $E_{2}^{(2)}$. Let us write $p_{m}^{k}=\boldsymbol{\lambda} E_{m}^{(k)}$. In the following we consider $k$ as a given positive integer, and to simplify notation, we drop the superscript $\left(^{k}\right)$ in $p_{m}^{k}$ and in $E_{m}^{(k)}$.

Since for each $m, E_{m}-E_{m+1} \subset C_{1}(m)$ and $T_{\mathrm{P}}\left(E_{m}-E_{m+1}\right)=E_{m+k}$, by (6) we have $\boldsymbol{\lambda} E_{m+k}=m \cdot \boldsymbol{\lambda}\left(E_{m}-E_{m+1}\right)$. That is to say, $p_{m+k}=$ $m\left(p_{m}-p_{m+1}\right)$, which rendered into homogeneous form gives

$$
p_{m+k}+m p_{m+1}-m p_{m}=0 .
$$

This is a finite difference equation of order $k$, linear with polynomial coefficients, which we will solve after establishing a few necessary lemmas.

Lemma 3. For all $m$,

$$
m p_{m}+p_{m+1}+p_{m+2}+\ldots+p_{m+k-1}=C, \quad C \text { constant } .
$$

Proof. Write the recurrence (8) as

$$
m p_{m}=m p_{m+1}+p_{m+k} .
$$

Use (9) recursively to get

$$
\begin{aligned}
m p_{m}+p_{m+1}+\ldots+p_{m+k-1} & =m p_{m+1}+p_{m+1}+\ldots+p_{m+k-1}+p_{m+k} \\
& =(m+1) p_{m+1}+p_{m+2}+\ldots+p_{m+k} .
\end{aligned}
$$

Thus, for all $m, m p_{m}+p_{m+1}+\ldots+p_{m+k-1}=C$.

LEMma 4. The sequence $p_{m}$ satisfies:

(a) $\lim _{m \rightarrow \infty} p_{m}=0$;

(b) $\lim _{m \rightarrow \infty} m p_{m}=1$.

Proof. Assertion (a) is trivial as $p_{m}=\boldsymbol{\lambda} E_{m}$ and $E_{m} \subset(0,1 / m]$. As for (b), if $q_{1}(x) \geq m$ and $x \notin E_{m}$, then there exists a place $j$ such that $q_{j}(x)=n$ and $q_{j+1}(x)<n+k$, that is, $x \in X_{m, n}$ for some $n$. By the covering rule,

$$
\boldsymbol{\lambda}\left(\left(0, \frac{1}{m}\right]-E_{m}\right) \leq \boldsymbol{\lambda} \bigcup_{n=m}^{\infty} X_{m, n} \leq \sum_{n=m}^{\infty} \boldsymbol{\lambda} X_{m, n} .
$$


By Lemma 1, this last sum is

$$
\sum_{n=m}^{\infty} \frac{1}{m}\left(\frac{1}{n+1}-\frac{1}{n+k}\right)=\frac{1}{m}\left(\frac{1}{m+1}+\ldots+\frac{1}{m+k-1}\right) .
$$

Consequently,

$$
p_{m} \geq \frac{1}{m}-\frac{1}{m}\left(\frac{1}{m+1}+\ldots+\frac{1}{m+k-1}\right),
$$

and (b) follows.

An immediate consequence of Lemma 4 is:

Lemma 5. The constant in Lemma 3 is 1 , that is, for all $m$ we have

$$
m p_{m}+p_{m+1}+p_{m+2}+\ldots+p_{m+k-1}=1 .
$$

For the particular values $m=2$ and $k=1$ the expression above gives $2 p_{2}=1$, that is, $\boldsymbol{\lambda} E_{2}^{(1)}=p_{2}=1 / 2$, which is result (ii) of Rényi's paper for Engel's series.

We now turn to solve the recurrence (8).

Proposition 1.

$$
p_{m}=C \int_{0}^{1} t^{m-1} e^{t+t^{2} / 2+\ldots+t^{k-1} /(k-1)} d t
$$

is a particular solution of the recurrence equation

$$
p_{m+k}+m p_{m+1}-m p_{m}=0,
$$

where $C$ is an arbitrary constant.

Proof. We will use Laplace's method (see [5, pp. 579-583]) which assumes that there exists a solution of the form

$$
p_{m}=\int_{a}^{b} t^{m-1} \omega(t) d t,
$$

where the limits $a$ and $b$ and the function $\omega(t)$ are to be determined. Using (12) and integrating by parts we get

$$
\begin{aligned}
p_{m+k} & =\int_{a}^{b} t^{m-1}\left(t^{k} \omega(t)\right) d t, \\
m p_{m} & =\left[t^{m} \omega(t)\right]_{a}^{b}-\int_{a}^{b} t^{m} \omega^{\prime}(t) d t, \\
m p_{m+1} & =\left[t^{m}(t \omega(t))\right]_{a}^{b}-\int_{a}^{b} t^{m}\left(\omega(t)+t \omega^{\prime}(t)\right) d t .
\end{aligned}
$$


Replacing all these expressions in the original equation (11) yields

$$
\begin{aligned}
\int_{a}^{b} t^{m-1}\left(t^{k} \omega(t)\right) d t+\left[t^{m}(t \omega(t))\right]_{a}^{b}-\int_{a}^{b} t^{m}\left(\omega(t)+t \omega^{\prime}(t)\right) d t & \\
-\left(\left[t^{m} \omega(t)\right]_{a}^{b}-\int_{a}^{b} t^{m} \omega^{\prime}(t) d t\right) & =0 .
\end{aligned}
$$

Grouping integrated parts and integrals we obtain

$$
\left[t^{m+1} \omega(t)-t^{m} \omega(t)\right]_{a}^{b}+\int_{a}^{b} t^{m-1}\left\{\left(t-t^{2}\right) \omega^{\prime}(t)+\left(t^{k}-t\right) \omega(t)\right\} d t=0 .
$$

The method is to annihilate the integrand in order to obtain $\omega(t)$ and to annihilate the bracketed part to determine the possible values of $a$ and $b$. We start with the integrand:

$$
\left(t-t^{2}\right) \omega^{\prime}(t)+\left(t^{k}-t\right) \omega(t)=0
$$

a linear homogeneous differential equation with separable variables,

$$
\frac{\omega^{\prime}}{\omega}=\frac{t-t^{k}}{t-t^{2}}=\frac{1-t^{k-1}}{1-t}=1+t+t^{2}+\ldots+t^{k-2},
$$

and thus

$$
\omega(t)=C e^{t+t^{2} / 2+\ldots+t^{k-1} /(k-1)},
$$

where $C$ is an arbitrary constant.

Now, replacing $\omega(t)$ with the value just found, we annihilate the integrated part seeking values $a$ and $b$ of $t$ that make

$$
t^{m} C e^{t+t^{2} / 2+\ldots+t^{k-1} /(k-1)}(t-1)=0 .
$$

Besides $\pm \infty$ depending on the parity of $k$, the only solutions are $a=0$ and $b=1$, which are precisely the values we are going to use to obtain a particular solution of our equation:

$$
p_{m}=C \int_{0}^{1} t^{m-1} e^{t+t^{2} / 2+\ldots+t^{k-1} /(k-1)} d t
$$

We are now ready for the main result of this section:

TheOREM 1. The measure of $E_{m}$ is

$$
p_{m}=e^{-(1+1 / 2+\ldots+1 /(k-1))} \int_{0}^{1} t^{m-1} e^{t+t^{2} / 2+\ldots+t^{k-1} /(k-1)} d t .
$$

Proof. By (10) from Lemma 5, we have the following set of initial conditions:

$$
m p_{m}+p_{m+1}+p_{m+2}+\ldots+p_{m+k-1}=1 \quad(m=1,2, \ldots) .
$$


Replacing $p_{m}$ gives

$$
C \int_{0}^{1}\left(m t^{m-1}+t^{m}+\ldots+t^{m+k-2}\right) e^{t+t^{2} / 2+\ldots+t^{k-1} /(k-1)} d t=1 .
$$

Since

$$
\begin{aligned}
\frac{d}{d t}\left\{t^{m} e^{t+t^{2} / 2+\ldots+t^{k-1} /(k-1)}\right\} & \\
& =\left(m t^{m-1}+t^{m}+\ldots+t^{m+k-2}\right) e^{t+t^{2} / 2+\ldots+t^{k-1} /(k-1)},
\end{aligned}
$$

we get

$C \int_{0}^{1}\left(m t^{m-1}+t^{m}+\ldots+t^{m+k-2}\right) e^{t+t^{2} / 2+\ldots+t^{k-1} /(k-1)} d t=e^{1+1 / 2+\ldots+1 /(k-1)}$.

Therefore, the infinite set of initial conditions above is consistent with the value of $C$ given by

$$
C=e^{-(1+1 / 2+\ldots+1 /(k-1))} .
$$

The solution for this particular $C$ is thus unique and corresponds to the measure we sought.

Now, as an immediate corollary of Theorem 1 , taking $m=1$ and $k=2$, and taking complements in $(0,1]$, we obtain $(7)$.

5. Conclusions. We establish the measure of different sets of real numbers in $(0,1]$ defined through properties satisfied by all the elements of their Pierce or Engel expansions. This settles the generalized version of a problem set by A. Rényi [9] concerning the measure of similar sets defined using Engel's series. We come out with quite nice and neat measures between zero and one. Specifically, replacing $m$ by 1 in Theorem 1 , the set $E_{1}^{(k)}$ of real numbers whose Pierce expansions present elements with a minimum jump of $k$ units has measure

$$
\boldsymbol{\lambda} E_{1}^{(k)}=e^{-\sum_{j=1}^{k-1} 1 / j} \int_{0}^{1} e^{\sum_{j=1}^{k-1} t^{j} / j} d t .
$$

\section{References}

[1] E. Borel, Sur les développements unitaires normaux, C. R. Acad. Sci. Paris 225 (1947), 773.

[2] P. Erdős, A. Rényi and P. Szüsz, On Engel's and Sylvester's series, Ann. Univ. Sci. Budapest. Sect. Math. 1 (1958), 7-32.

[3] J. Galambos, Representations of Real Numbers by Infinite Series, Lecture Notes in Math. 502, Springer, Berlin, 1976. 
[4] J. Galambos, The Asymptotic Theory of Extreme Order Statistics, Wiley, New York, 1978; 2nd ed.: Robert Krieger, Malabar, FL, 1987.

[5] K. Jordan, Calculus of Finite Differences, 3rd ed., Chelsea, New York, 1979. (First ed. Budapest, 1939).

[6] P. Lévy, Remarques sur un théorème de M. Émile Borel, C. R. Acad. Sci. Paris 225 (1947), 918-919.

[7] J. Paradís, Ll. Bibiloni and P. Viader, On actually computable bijections between $\mathbb{N}$ and $\mathbb{Q}^{+}$, Order 13 (1996), 369-377.

[8] J. Paradís, P. Viader and Ll. Bibiloni, Approximation of irrational quadratics and their Pierce expansions, Fibonacci Quart. 36 (1998), 146-153.

[9] A. Rényi, A new approach to the theory of Engel's series, Ann. Univ. Sci. Budapest Sect. Math. 5 (1962), 25-32.

[10] F. Schweiger, Ergodic Theory of Fibred Systems and Metric Number Theory, Clarendon Press, Oxford, 1995.

[11] J. O. Shallit, Some predictable Pierce expansions, Fibonacci Quart. 22 (1984), $332-335$.

[12] - Metric theory of Pierce expansions, ibid. 24 (1986), 22-40.

[13] - Pierce expansions and rules for the determination of leap years, ibid. 32 (1994), 416-423.

[14] W. Sierpiński, Sur quelques algorithmes pour développer les nombres réels en série, C. R. Soc. Sci. Varsovie 4 (1911), 56-77 (in Polish). A French translation can be found in Oeuvres choisies, T. I, Varsovie, 1974, 236-254.

[15] D. Williams, On Rényi's 'record' problem and Engel's series, Bull. London Math. Soc. 5 (1973), 235-237.

Applied Mathematics

Departament d'Economia i Empresa

Univ. Pompeu Fabra

c/ Ramon Trias Fargas 25-27

08005 Barcelona, Spain

E-mail: pelegri.viader@econ.upf.es

jaume.paradis@econ.upf.es
Facultat de Ciències de l'Educació

Univ. Autònoma de Barcelona 08193 Bellaterra, Barcelona, Spain E-mail: 1.bibiloni@uab.es 